\title{
Mental health research trends in Sri Lanka
}

\author{
S S Williams ${ }^{1}$, S N Hewage ${ }^{2}$ and I R Karandawala ${ }^{3}$
}

(Index words: psychiatric research, research activities, statistics, bibliometrics)

\begin{abstract}
Objective To review the number of research publications in the field of mental health in Sri Lanka from 1900 to 2009.

Methods A search of all publications in psychiatry and mental health from Sri Lanka was conducted using Pubmed, all medical journals published in Sri Lanka and researchers. The identified papers were reviewed for their content and categorised as research in psychiatry and mental health, based on strict inclusion and exclusion criteria.
\end{abstract}

Results A total of 207 papers were identified. The first mental health research publication from Sri Lanka is in 1964. The last decade (2000-2009) accounted for $62 \%$ of the publications with the majority of the papers being published in indexed journals. The Ceylon Medical Journal carried the most number of papers and the topic on which most of the research was conducted was suicide and deliberate self harm.

Conclusions There is an increasing trend towards research in to psychiatry and mental health in Sri Lanka.

Ceylon Medical Journal 2011; 56: 61-65

\section{Introduction}

Good quality research is vital to promote scientific rigor in the practice of medicine particularly in this era of evidence based medicine (EBM) [1,2]. In this sense, research into mental health and psychiatry is essential for the development of psychiatry in Sri Lanka.

Most of the evidence is currently obtained from studies conducted in high income countries particularly in the west, where the population in which the research is conducted has vastly different ethnic, socio-cultural and demographic characteristics from our own. Many of these research findings are not directly applicable to our local context.

Psychiatry is a branch of medicine where nonbiological factors have a significant impact on diagnosis and treatment outcome. Hence, there is all the more reason to focus on promoting research that is locally relevant. Trends, however, in relation to research output in mental health and psychiatry in Sri Lanka, and the areas researched or under-researched, are not known.

The objective of this study was to conduct a retrospective review of the research output in Sri Lanka on mental health from 1900 up to the end of year 2009, and to identify the main areas in which the research has been conducted.

\section{Methods}

A search of all published research in mental health and psychiatry in Sri Lanka from 1st January 1900 to 31st December 2009 was conducted. An internet search was conducted using search engines such as Pubmed looking

${ }^{1}$ Department of Psychiatry and ${ }^{2}$ Medical Education Centre, Faculty of Medicine, University of Kelaniya, and ${ }^{3}$ Department of Psychiatry, Faculty of Medical Sciences, University of Sri Jayawardenapura Sri Lanka.

Correspondence: SS, e-mail <shehan.williams@linacre-oxford.com>. Received 1 October 2010 and revised version accepted 15 February 2011. Competing interests: none declared. 
for the terms 'Sri Lanka', 'Ceylon', 'mental health' and 'psychiatry' and the Sri Lanka journals online portal. All local medical journals and medical bibliographies were hand searched. Local psychiatrists, psychologists, mental health professionals and researchers were notified of our attempts to locate publications and requested to send their publications. The authors also had access to a previously published bibliography of research in to mental health and psychiatry in Sri Lanka [3].

The content was thereafter assessed based on their relevance to Sri Lanka. Each individual article was scrutinised by three experts (two consultant psychiatrists and a research medical officer), and was included if they met the following criteria: containing research data including case reports on psychiatry/mental health and the research was conducted in Sri Lanka and/or on a Sri Lankan population.

This literature was further divided into indexed journals and those from non indexed journals. Articles which could not be clearly categorised as research in psychiatry or mental health but were related topics such as sociology or anthropology (eg: smoking, intimate partner violence) and those that were not research but expert opinion on varying topics were excluded. The flow chart of the process is shown in Figure 1.

The inclusion and exclusion criteria did not take into account the quality of the research. A further attempt was made to categorise the research into the International Classification of Diseases (ICD 10) categories, subspecialties and common themes.

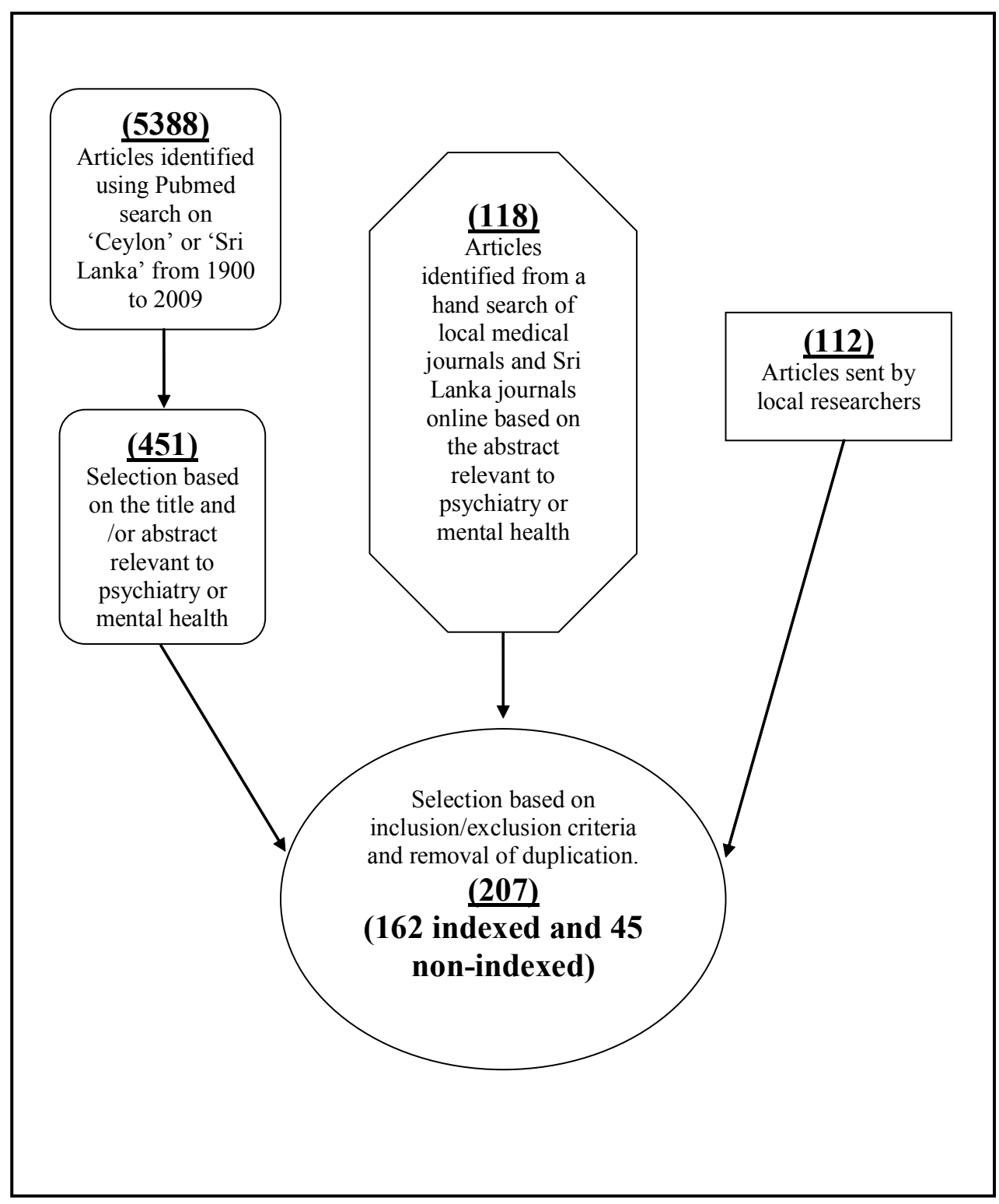

Figure 1. Flow chart of review process. 


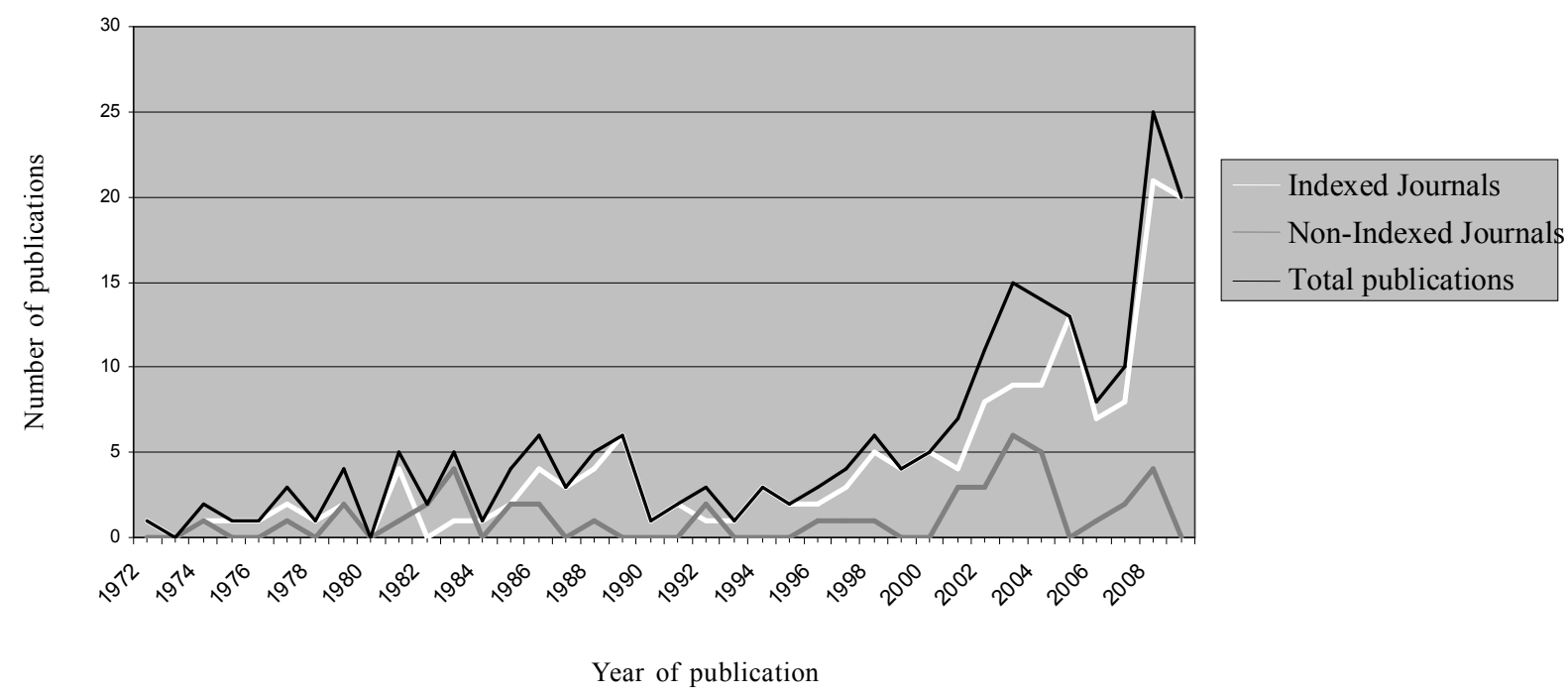

Figure 2. The temporal trend in research publications on mental health in Sri Lanka.

Table 1. Frequency of published papers according to five year periods

\begin{tabular}{|c|c|c|c|}
\hline \multirow{2}{*}{$\begin{array}{l}\text { Five year period } \\
\text { of publication }\end{array}$} & \multicolumn{2}{|c|}{ Journal type } & \multirow[t]{2}{*}{ Total } \\
\hline & Indexed journals & Non-indexed journals & \\
\hline 1970-1974 & $2(1.2 \%)$ & $1(2.2 \%)$ & $3(1.4 \%)$ \\
\hline 1975-1979 & $7(4.3 \%)$ & $3(6.7 \%)$ & $10(4.8 \%)$ \\
\hline 1980-1984 & $6(3.7 \%)$ & $7(15.6 \%)$ & $13(6.3 \%)$ \\
\hline 1985-1989 & $19(11.7 \%)$ & $5(11.1 \%)$ & $24(11.6 \%)$ \\
\hline 1990-1994 & $8(4.9 \%)$ & $2(4.4 \%)$ & $10(4.8 \%)$ \\
\hline 1995-1999 & $16(9.9 \%)$ & $3(6.7 \%)$ & $19(9.2 \%)$ \\
\hline 2000-2004 & $35(21.6 \%)$ & $17(37.8 \%)$ & $52(25.1 \%)$ \\
\hline 2005-2009 & $69(42.6 \%)$ & $7(15.6 \%)$ & $76(36.7 \%)$ \\
\hline Total & $162(100.0 \%)$ & $45(100.0 \%)$ & $207(100.0 \%)$ \\
\hline
\end{tabular}

Table 2. Indexed journals with three or more research papers on mental health from Sri Lanka

\begin{tabular}{lc}
\hline Journal name & Frequency (\%) \\
\hline Ceylon Medical Journal & $39(24.1 \%)$ \\
British Journal of Psychiatry & $10(6.2 \%)$ \\
Acta Psychiatrica Scandinavica & $8(4.9 \%)$ \\
Social Science and Medicine & $6(3.7 \%)$ \\
International Journal of Social Psychiatry & $4(2.5 \%)$ \\
Social Psychiatry and Psychiatric Epidemiology & $4(2.5 \%)$ \\
Alcohol and Alcoholism & $3(1.9 \%)$ \\
BMC Psychiatry & $3(1.9 \%)$ \\
BMC Public Health & $3(1.9 \%)$ \\
Bulletin of the World Health Organization & $3(1.9 \%)$ \\
Child Abuse and Neglect & $3(1.9 \%)$ \\
International Journal of Geriatric Psychiatry & $3(1.9 \%)$ \\
Psychological Medicine & $3(1.9 \%)$ \\
\hline
\end{tabular}


Table 3. Non-indexed journals with three or more research papers on mental health from Sri Lanka

\begin{tabular}{lc}
\hline Journal name & Frequency (\%) \\
\hline Sri Lanka Journal of Child Health (formerly Ceylon Journal of Child Health) & $13(28.9 \%)$ \\
Jaffna Medical Journal & $7(15.6 \%)$ \\
Journal of the Ceylon College of Physicians & $7(15.6 \%)$ \\
Galle Medical Journal & $5(11.1 \%)$ \\
Sri Lankan Family Physician & $3(6.7 \%)$ \\
\hline
\end{tabular}

\section{Results}

A total of 207 papers met the inclusion criteria. Of these, $162(78.3 \%)$ were in indexed journals and $45(21.7 \%)$ were in non indexed journals. The first identified paper was in 1964. There were $13(6.3 \%)$ papers between 1970 and 1977; 37 (17.9\%) papers between 1980 and 1989; 29 (14\%) papers between 1990 and 1999, and 128 (61.8\%) papers between 2000 and 2009 (Table 1 and Figure 2)

The Ceylon Medical Journal had the most number of papers $(39 ; 18.8 \%$ ) followed by Sri Lanka Journal of Child Health $(13 ; 6.3 \%)$ and British Journal of Psychiatry $(10 ; 4.8 \%)$ (Tables 2 and 3$)$. A total of 71 indexed journals and 13 non-indexed journals carried the papers.

Most of the research was conducted in relation to general adult psychiatry (97), with 44 on children and adolescents, 15 on the elderly and 14 on addiction. Two studies each in psychotherapy and learning disability and one study in forensic psychiatry had been conducted.

Suicide and deliberate self harm accounted for the most number of studies on a single topic with 44 publications on this topic. Other topics in order of frequency are - war and conflict - 19; tsunami - 10 and culture bound syndromes -8 . There were 14 studies on epidemiology, 10 on validation of mental health scales and instruments, 9 on mental health services and 5 on clinical trials. There were 22 case reports. Only 72 papers could be fitted in to ICD 10 categories as the others had non-specific psychiatric diagnoses or multiple diagnostic categories.

\section{Discussion}

Our findings show an increase in research publications on mental health in Sri Lanka over time. The majority of research publications have been in the last decade (2000-2009) - an increase of more than 200\% from the previous decades.

It is also heartening to note that the majority of the publications are in indexed journals. The paucity of research in psychiatry from low and middle income countries published in high impact journals has been highlighted before [4,5]. Publishing in indexed journals, no doubt provides wider access to these research findings. The 10/90 report on health research argues that less than
$10 \%$ of the world's research resources are earmarked for $90 \%$ of the health problems [6]. The enormous inequalities in global health research is shown by the underrepresentation of publications and authors from developing countries in high impact medical journals [7]. Our study did not however, look at the contribution to these papers by local and foreign authors and the extent of collaboration.

The most number of papers on research in psychiatry in Sri Lanka have been published in the Ceylon Medical Journal. Studies published in this journal are widely read by local clinicians and are also indexed for Pubmed, thus providing international access. This is certainly an incentive to publish local research in the Ceylon Medical Journal.

The research however has been conducted mainly in a few areas of interest. On top of the list is suicide and deliberate self harm, with war and conflict related research coming second. Rightly, the focus has been on suicide and deliberate self harm as it has been high in Sri Lanka in the late 80 s and early 90s [8]. The war and tsunami have been other significant events in the past decade. Resources were probably allocated for research in to these areas in the past few years. It is prudent now however to allocate resources to other identified research priorities.

The research agenda is low within the government health sector as in many other low and middle income countries, where competing service needs take priority [9]. The mental health research mapping group that looked at research in the South Asian region found that important criteria for prioritising research were burden of disease, social justice, and availability of funds [10]. The burden of mental illness is hidden, and therefore ignored by funding agencies and policy makers. It is projected that the burden of mental illness in 2020 will be the highest in comparison to all other diseases, in terms of disability adjusted life years (DALYs) [11].

Few clinical trials have been conducted. Clinical trials are essential to generate the evidence that can be applied to clinical practice. Collaboration and a culture of sound ethical standards will have to be developed for the conduct of experimental studies [12]. Research involving psychiatric subjects with doubtful capacity has to be regulated and the horrors of the past cannot ever be repeated $[13,14]$. 
The attempt to classify the papers into ICD categories was fraught with difficulty. Many papers could not be fitted to an ICD category. Others used vague categorisation of psychological states and behaviour. A factor contributing to the lack of this categorisation maybe the lack of validated psychometric and diagnostic tools in the local languages [15]. Much effort has to be directed at validating psychometric tools which can be applied in research.

A possible limitation of this search would be the possibility of missing research published in non-indexed journals in other countries including India. However, most studies with local authors would have been included as an attempt was made to contact all potential local authors for a list of their publications. Studies prior to 1900 are difficult to trace as journals from this era are usually not indexed and are not available in libraries.

The reasons for the decline in publications in 19901999 in relation to 1980-1989 are not clear. Whether the socio-political climate at that time and the migration of mental health professionals to other countries contributed to this phenomenon is a matter for debate. The increase in publications in the last decade would no doubt have been facilitated by the access to electronic communication. It is hoped that the current decade -2010 to 2019 , will continue with the research momentum seen in the last decade. Research should not decline in this post tsunami and conflict era. It should now focus on meeting the research needs of the country and the region.

\section{References}

1. Claridge JA, Fabian TC. History and development of evidence-based medicine. World Journal of Surgery 2005; 29: 547-53.

2. Sackett DL, Rosenberg WM, Gray JA, Haynes RB, Richardson WS. Evidence based medicine: what it is and what it isn't. British Medical Journal 1996; 312: 71-2.

3. Karandawela I, Williams S, Hewage S. Bibliography of Mental Health Research in Sri Lanka. Sri Lanka College of Psychiatrists, 2009.

4. Patel V, Sumathipala A. International representation in psychiatric literature: survey of six leading journals. British Journal of Psychiatry 2001; 178: 406-9.
5. Patel V, Kim YR. Contribution of low- and middle-income countries to research published in leading general psychiatry journals, 2002-2004. British Journal of Psychiatry 2007; 190: $77-8$.

6. Global Forum for Health Research. The 10/90 report on health research. Geneva: Global Forum for Health Research, 2000.

7. Sumathipala A, Siribaddana S, Patel V. Under-representation of developing countries in the research literature: ethical issues arising from a survey of five leading medical journals. BMC Medical Ethics 2004; 5: E5.

8. World Health Organization. Mental Health. Suicide prevention. Available from: http://www.who.int/ mental_health/media/sril.pdf .[accessed 25 January 2011]

9. Razzouk D, Sharan P, Gallo C, et al. WHO-Global Forum for Health Research Mental Health Research Mapping Project Group. Scarcity and inequity of mental health research resources in low- and middle-income countries: a global survey. Health Policy 2010; 94: 211-20.

10. Sharan P, Gallo C, Gureje O, et al. World Health Organization-Global Forum for Health Research - Mental Health Research Mapping Project Group. Mental health research priorities in low- and middle-income countries of Africa, Asia, Latin America and the Caribbean. British Journal of Psychiatry 2009; 195: 354-63.

11. World health Organisation. Chapter 2: Burden of Mental and Behavioural Disorders in The World Health Report 2001 - Mental Health: New Understanding, New Hope. WHO Geneva 2001: 19-45

12. Ballantyne AJ. How to do research fairly in an unjust world. American Journal of Bioethics 2010; 10: 26-35.

13. Ghias K, Ahmer S. Guarding the guardians: bioethics curricula for psychiatrists-in-training in developing countries. International Review of Psychiatry 2010; 22: 294-300.

14. Kerpel-Fronius S. Influence of the Nuremberg physicians' trials - beginning a new era in the ethical judging of human experiments. Orvosi Hetilap 2008; 149: 195-201.

15. Kuruppuarachchi KALA, Williams SS. Cross-cultural psychiatric interviews and research instruments (Correspondence). British Journal of Psychiatry 2001; 179: 461. 